\title{
Swallowing disorder following salvage total pharyngo-laryngo-esophagectomy with free jejunum reconstruction
}

\author{
TAKASHI MARUO ${ }^{1}$, MINORU SAKURABA ${ }^{2}$, TAKESHI SHINOZAKI ${ }^{3}$, TOSHIFUMI TOMIOKA ${ }^{3}$, \\ WATARU OKANO ${ }^{3}$, SATOKO ARAHIRA $^{4}$, YOSHIE IINO ${ }^{5}$ and RYUICHI HAYASHI ${ }^{3}$ \\ ${ }^{1}$ Department of Otorhinolaryngology, Nagoya University, Nagoya, Aichi 466-8550; \\ ${ }^{2}$ Department of Plastic Surgery, Iwate Medical School, Morioka, Iwate 020-8505; \\ Departments of ${ }^{3} \mathrm{Head}$ and Neck Surgery, ${ }^{4}$ Radiation Oncology and ${ }^{5}$ Rehabilitation, \\ National Cancer Research Center Hospital East, Kashiwa, Chiba 277-0882, Japan
}

Received October 18, 2017; Accepted January 25, 2018

DOI: $10.3892 / \mathrm{ol} .2018 .8213$

\begin{abstract}
For recurrent cases or residual cases following concomitant chemo-radiation therapy (CCRT), salvage surgery is a frequently used treatment options. A swallowing disorder is one of the major complications of CCRT. The purpose of the present study was to evaluate the effect of CCRT on swallowing function in patients who underwent salvage total pharyngo-laryngo-esophagectomy (TPLE), and to evaluate the importance of pharyngeal constriction in patients who underwent TPLE. Between 2008 January and 2014 May, 54 patients were treated with salvage TPLE following CCRT or TPLE at the National Cancer Center Hospital East, Chiba, Japan and were included in the present study. A total of 14 patients underwent salvage TPLE following CCRT for recurrence or residual tumor (the salvage TPLE group), and 40 patients underwent TPLE as initial treatment (the TPLE group). The pharyngeal constriction score and the post-swallowing oropharyngeal residue rate were evaluated, and inadequate velopharyngeal closure was assessed by videofluorography. The pharyngeal constriction score of the salvage TPLE group was poorer than that of the TPLE group $(\mathrm{P}<0.05)$. The bolus residue in the oropharynx was significantly larger in the salvage TPLE group than in the TPLE group $(\mathrm{P}<0.05)$. With regards to inadequate velopharyngeal closure, there was no significant difference between the TPLE group and the salvage TPLE group $(\mathrm{P}>0.99)$. The results of the present study indicate that the swallowing function of patients who undergo salvage TPLE may be affected by CCRT.
\end{abstract}

Correspondence to: Dr Takashi Maruo, Department of Otorhinolaryngology, Nagoya University, 65 Tsurumai, Nagoya, Aichi 466-8550, Japan

E-mail: tmaruo@med.nagoya-u.ac.jp

Keywords: salvage surgery, total pharyngo-laryngo-esophagectomy, dysphagia, pharyngeal construction, chemoradiotherapy

\section{Introduction}

Concomitant chemo-radiation therapy (CCRT) is a standard treatment for head and neck cancer $(1,2)$. For recurrent cases or residual cases following CCRT, salvage surgery is one of the important treatment options (3). With the increasing use of CCRT in the treatment of head and neck cancer (4), salvage surgery following failed CCRT will be increasingly prominent. Higher rates of acute and long-term toxicity following CCRT could result in higher rates of postoperative morbidity and mortality $(5,6)$. Swallowing disorders are major complications of CCRT that can directly affect the quality of life of the patient. Previous studies have shown that the major complications of CCRT are xerostomia, reduced mobility of the tongue base, reduced mobility of the larynx, reduced pharyngeal and laryngeal sensation (incomplete protection of the airway) and trismus $(7,8)$. These complications can result in the reduction of pharyngeal construction and inadequate laryngeal closure. Pharyngeal constriction, which presses the bolus to the esophagus, and laryngeal closure, which prevents bolus invasion to the lungs, serve major roles in swallowing function. In this sense, it seems that total laryngectomy and total pharyngo-laryngo-esophagectomy (TPLE) with free jejunal graft reconstruction cannot cause dysphagia because the larynx is removed. However, the incidence of dysphagia following total laryngectomy has been reported to range between 10 and $60 \%$ (9). Similarly, the overall reported incidence of dysphagia following pharyngolaryngectomy with free jejunal graft reconstruction is reported to range between 2 and 58\% (10). Previous studies $(9,10)$ have reported a higher incidence of dysphagia at discharge and at long-term follow-up in patients that underwent laryngectomy or pharyngolaryngectomy. We therefore hypothesized that pharyngeal constriction is strongly associated with swallowing function following TPLE. Furthermore, swallowing function following salvage TPLE is poorer than that following TPLE as an initial treatment, because the pharyngeal membrane of the patients who received salvage TPLE has already been affected by initial CCRT. To the best of our knowledge, there have been 
no reports concerning dysphagia following salvage surgery, particularly salvage TPLE.

The purpose of the present study was to evaluate the influence of CCRT on pharyngeal constriction by comparing swallowing function between the salvage TPLE group and the initial TPLE group, and to confirm the role of pharyngeal constriction following TPLE.

\section{Materials and methods}

Study design and patients. The present study was a retrospective study conducted in a single institute in the National Cancer Research Center Hospital East (Kashiwa, Japan). The present study was approved by the Research Ethics Committee of the National Cancer Research Center Hospital East, and the research outline is open to the public.

Eligible patients were those who received salvage TPLE following CCRT or received TPLE as initial treatment for hypopharyngeal cancer, those whose clinical records were available and those who presented for treatment between 2008 January and 2014 May in the National Cancer Research Center Hospital East. CCRT in the current study was defined as at least one course of chemotherapy with $>60$-Gy radiation.

A total of 212 patients underwent TPLE with free jejunal reconstruction for laryngeal cancer and hypopharyngeal cancer between 2008 January and 2014 May in the National Cancer Research Center Hospital East, Chiba, Japan. A total of 60 patients were eligible for the current study, with the remaining patients excluded because they lacked videofluorography (VF) records. A further 6 of these 60 patients with laryngeal cancer: Glottic cancer and subglottic cancer, were excluded as the range of radiotherapy for glottic cancer and subglottic cancer was different from hypopharyngeal cancer and supraglottic cancer; thus, 54 patients (48 males, 6 females; median age 66.3 years; age range, $36-81$ years) with hypopharyngeal cancer were included in the present study. Overall, 14 patients received salvage TPLE following CCRT for recurrence or residual tumor (the salvage TPLE group), and 40 patients received TPLE as initial treatment (the TPLE group) (Table I). The TNM stage of eligible patients was defined by Union Against Cancer and American Joint Committee on Cancer staging system for head and neck cancer, seventh edition (11).

Chemoradiotherapy. Chemoradiotherapy was performed for all patients in the salvage TPLE group. The chemotherapy regimens are depicted in Table II.

Following CT simulation in the treatment position, the radiation dose plan was made for each individual patient. A conventional fractionation schedule of 2 Gy/day was used. Only patients who received a total radiation dose that was $>60$ Gy were included in this study. The field of radiation is shown in Fig. 1.

Surgery. All surgery was performed at the National Cancer Research Center Hospital East by a single surgical team. All patients in the present study received TPLE with free jejunal reconstruction. With regards to primary resection, the superior margin was set based on the degree of cancer progression. For 40 patients, the superior margin was set to over the hyoid bone,
Table I. Chemotherapy regimens.

\begin{tabular}{lc}
\hline Regimen & Patients, $\mathrm{n}$ \\
\hline CDDP & 11 \\
Cetuximab & 2 \\
5-FU + CDDP & 3 \\
Total & 16 \\
\hline
\end{tabular}

CDDP, cisplatin; 5-FU, 5-fluorouracil.

Table II. Patient characteristics $(n=60)$.

\begin{tabular}{lrc}
\hline Characteristic & TPLE, $\mathrm{n}$ & Salvage TPLE, $\mathrm{n}$ \\
\hline Total & 44 & 16 \\
Sex & & \\
Male & 40 & 14 \\
Female & 4 & 2 \\
Tumor site & & \\
Hypopharynx & 40 & 14 \\
Larynx (supraglottic) & 4 & 2 \\
Clinical T classification & & \\
T2 & 8 & 2 \\
T3 & 9 & 7 \\
T4a & 25 & 4 \\
T4b & 2 & 1 \\
Clinical N classification & & \\
N0 & 11 & 6 \\
N1 & 6 & 2 \\
N2 & 24 & 0 \\
N3 & 3 & 1 \\
Combined resection of the & 13 & \\
oropharynx & & \\
& &
\end{tabular}

${ }^{a}$ The superior margin is set to the tonsils. TPLE, total pharyngo-laryngo-esophagectomy; T, tumor; N, node (11).

and for the remaining 14 patients, it was set to the tonsil. The anal side margin was also set based on cancer progression, and it was set at the same level as tracheostomy for all patients. No patients exhibited a tumor extension to an area lower than cervical esophagus, and no patients required mediastinal tracheostomy.

Level 2-4 neck dissection (ND) was performed for the bilateral neck in the TPLE group. In the salvage TPLE group, ND was not performed. Retropharyngeal lymph node dissection was not performed for either group.

Reconstruction surgery. Following jejunal trimming on the oral side, pharyngojejunostomy was performed according to the Gambee technique (12), with 4-0 absorbable monofilament sutures. The anal side of the jejunal conduit was then trimmed 


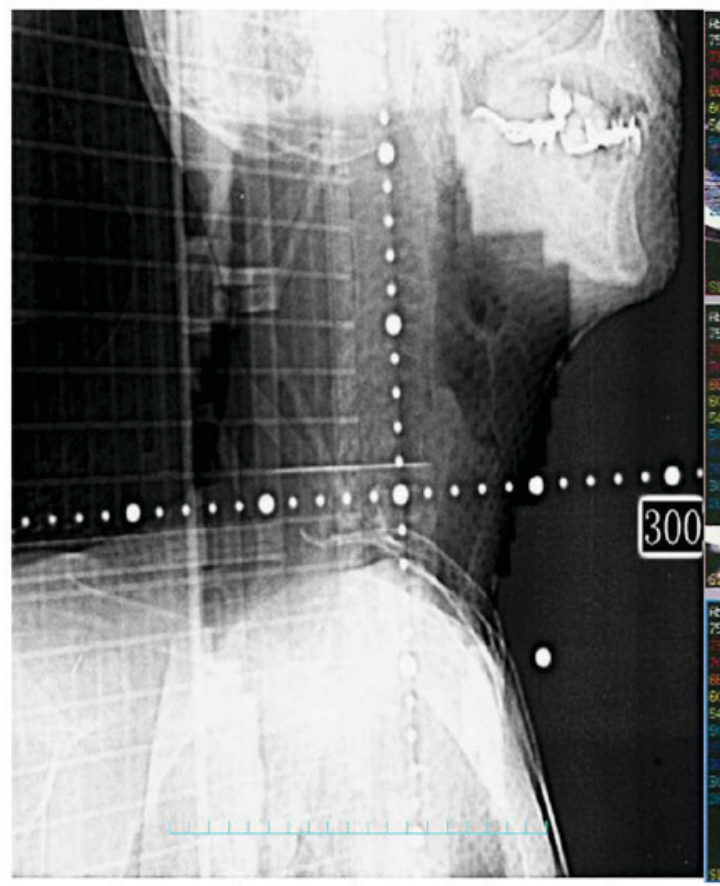

Laryngeal cancer (supraglottic cancer)
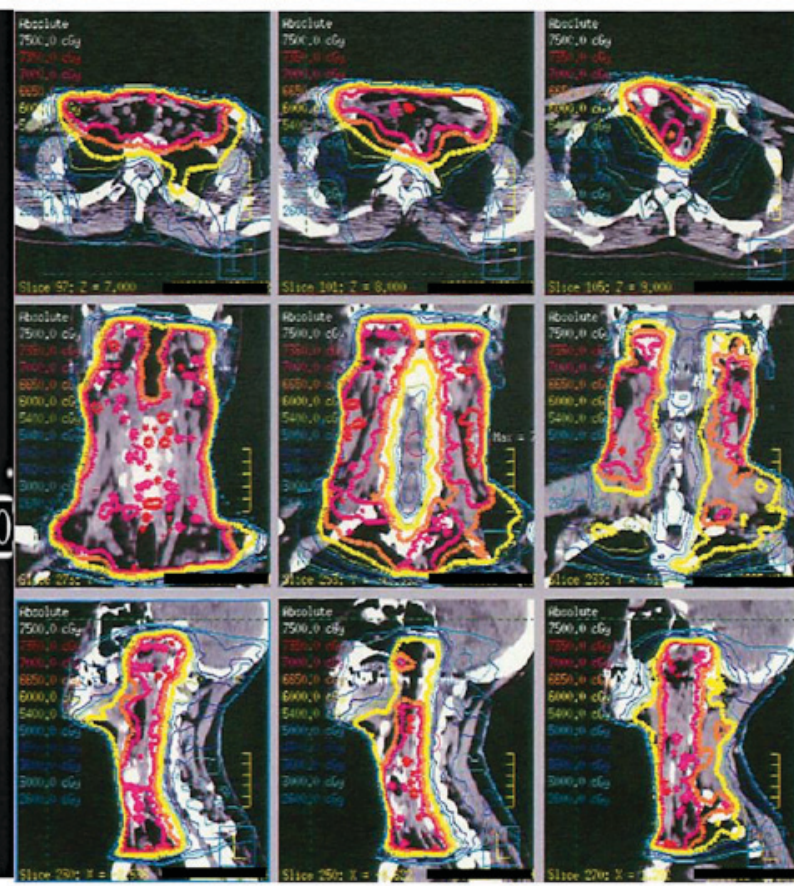

Hypopharyngeal cancer (IMRT)

Figure 1. Radiation range for laryngeal cancer and hypopharyngeal cancer. The radiation range is approximately the same for all patients who received chemoradiation. The most cranial area of the radiation range is the top of the oropharynx, and the caudal end of the radiation range is the supraclavicular area.

so that the jejunal graft would be pulled straight following complete enteric anastomosis. When relaxed, the trimmed jejunum was approximately two-thirds the length of the defect. Jejunoesophagostomy was performed using the Gambee technique, using 4-0 absorbable monofilament sutures. Following complete enteric anastomosis, the microscopic vascular anastomoses were established (13).

Swallowing assessment. Swallowing was assessed by VF using a modified barium swallow procedure (MBS). The contrast medium was $40 \%$ barium sulfate, and a digital video recorder was used to record the images observed from the lateral and frontal perspectives (30 frames/sec). The MBS protocol included swallowing boluses of $5 \mathrm{ml}$ of thin liquid barium. Postoperative VF examination was performed at 7 days after surgery in the TPLE group and at 14 days in the salvage TPLE group.

Swallowing function was evaluated using the pharyngeal constriction score (14), the post-swallow pharyngeal residue rate and velopharyngeal regurgitation on VF. Pharyngeal constriction has been proposed as a parameter to distinguish functional from impared swallows (15-19). The parameters for evaluation of swallowing function were then determined, and those parameters were measured at the first swallow in the first VF study following surgery by one investigator. The first swallow was used to measure the parameters more easily than the second and subsequent swallows, because there was no bolus residue remaining from the previous examination.

With regards to the pharyngeal constriction score, the pharyngeal constriction ratio (PCR) is a well-established tool for measuring and monitoring pharyngeal constriction (16). It has been validated as a surrogate measure of strength and is associated with manometric findings $(17,20)$. In the present study, the pharyngeal constriction score was determined using the VF evaluation criteria of the Japanese Society of Dysphagia Rehabilitation (14). This score is based on the data from VF analysis. The pharyngeal constriction score ranges from 1 to 3 , with a higher score indicating a better contact of the front and back of the pharynx. Score 3 is normal (complete contact of the front and back and elimination of the air space of the pharynx); score 2 is inadequate contact of the front and back of the pharynx; and score 1 is abnormal (no contact of the front and back of the pharynx). In this analysis, a score of 3 was defined as normal, and a score of 2 or 1 demonstrated that patients had constriction disorder. VF finding criteria that are matched with the pharyngeal constriction score are depicted in Fig. 2. The pharyngeal score was estimated based on these criteria.

With regards to the post-swallow oropharyngeal residue rate, the residue rate was defined as the proportion of the oropharyngeal residue of the total bolus. The proportion of the oropharyngeal residue was estimated by comparing the thinness of the bolus in the oropharynx prior to and following swallowing using the following formula: Post-swallowing oropharyngeal residue rate $=$ Oral-pharyngeal residue/total bolus x100 (\%) (Fig. 3). The volume of residue was measured in the same way as the oropharyngeal swallow efficiency (OPSE). OPSE is a global measure of swallowing function used to quantify the interaction between the speed of bolus movement and the safety/efficiency of the mechanism in clearing material from the oropharynx (21).

With regards to inadequate velopharyngeal closure, the bolus flowing backward in the velopharynx was defined as a positive finding. 


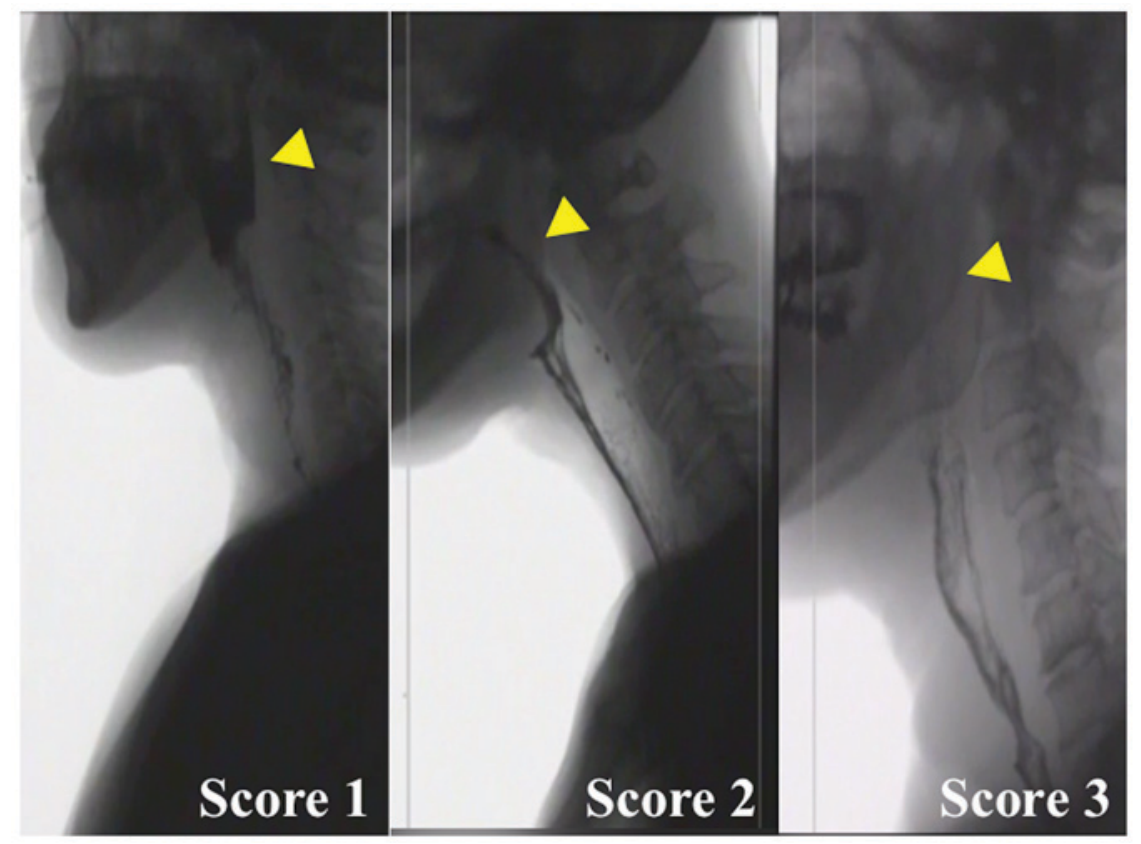

Figure 2. The pharyngeal constriction score from the videofluorography evaluation criteria of the Japanese Society of Dysphagia Rehabilitation. Score 3 , Normal (complete contact of the front and back and elimination of the air space of the pharynx); score 2, inadequate contact of the front and back of the pharynx; score 1, abnormal (no contact of the front and back of the pharynx). A score of 3 is normal, and a score of 2 or 1 shows that patients have constriction disorder.

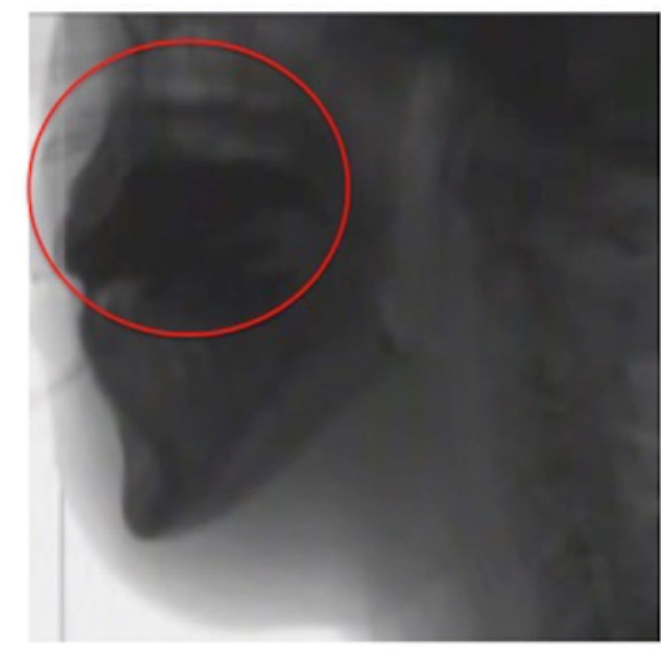

Before swallow

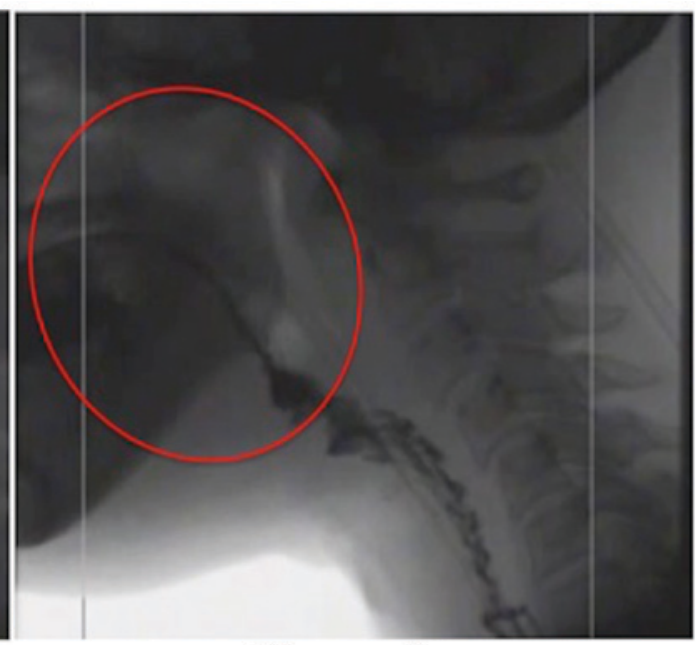

After swallow Oropharyngeal residue

Figure 3. The volume of oropharyngeal residue is defined as the residual bolus volume in the oral and pharyngeal space after swallowing. The total volume is the bolus volume in the oral phase prior to swallowing.

Statistical analysis. Data were analyzed to evaluate differences between the salvage TPLE group and the TPLE group using Fisher's exact test, Wilcoxon rank sum test and Pearson's $\chi$ test, as appropriate. $\mathrm{P}<0.05$ was considered to indicate a statistically significant difference. All analyses were performed using JMP 11 (SAS Institute, Inc., Cary, NC, USA).

\section{Results}

Pharyngeal constriction score. The pharyngeal constriction scores are depicted in Table III; this score is defined in
Fig. 2, and a high score is indicative of good constriction. In the TPLE group, 35 patients had a score of 3 and 9 patients had a score of 2 ; no patients had a score of 1 . In the salvage TPLE group, 8 patients had a score of 3,5 patients had a score of 2 , and 3 patients had a score of 1 . Scores of 2 and 1 were defined as inadequate pharyngeal constriction, and a score of 3 was defined as normal constriction. Inadequate pharyngeal constriction was observed in 9 patients in the TPLE group and 8 patients in the salvage TPLE group. Normal pharyngeal constriction was observed in 35 patients in the TPLE group and 8 patients in the salvage TPLE group. Using a Pearson's 
Table III. Pharyngeal constriction scores by group.

\begin{tabular}{lcc}
\hline Score & TPLE, $\mathrm{n}$ & Salvage TPLE, $\mathrm{n}$ \\
\hline 3 & 35 & 8 \\
2 & 9 & 5 \\
1 & 0 & 3 \\
\hline
\end{tabular}

TPLE, total pharyngo-laryngo-esophagectomy.

Table IV. Comparison of pharyngeal constriction.

\begin{tabular}{lccc}
\hline Group & Inadequate, $\mathrm{n}$ & Normal, $\mathrm{n}$ & P-value \\
\hline TPLE & 9 & 35 & $0.0247^{\mathrm{a}}$ \\
Salvage TPLE & 8 & 8 & \\
Total & 17 & 43 & \\
\hline
\end{tabular}

aTPLE group vs. salvage TPLE group. TPLE, total pharyngo-laryngo-esophagectomy.

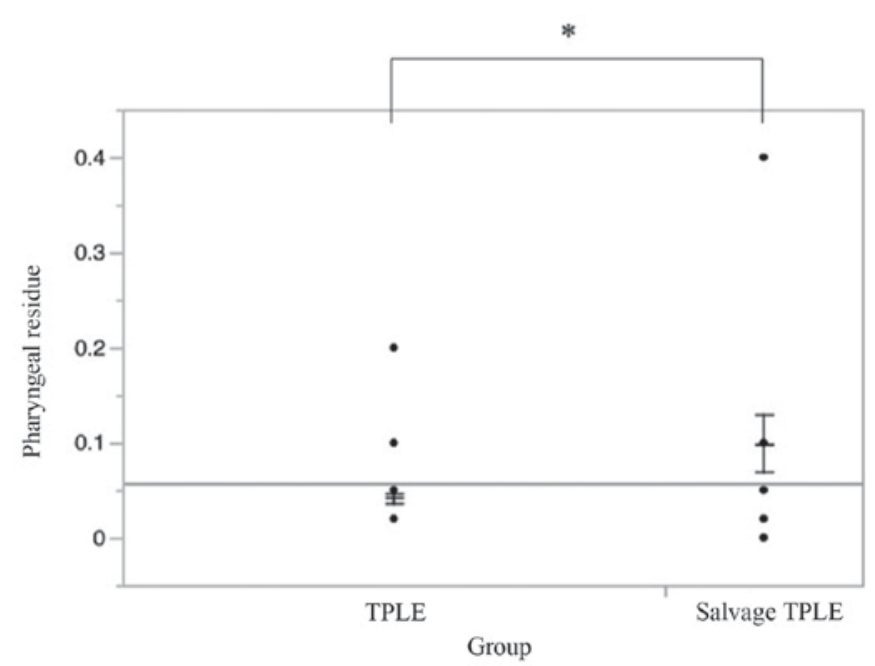

Figure 4. Comparison of pharyngeal residue between TPLE and salvage TPLE. The vertical axis depicts the residual rate and the horizontal axis depicts each groups. A plot of the same residual rate is overlaid. ${ }^{*} \mathrm{P}<0.05$. TPLE, total pharyngo-laryngo-esophagectomy.

$\chi^{2}$ test, the association between pharyngeal constriction type (normal or inadequate) and surgery type (salvage or initial) was investigated. There was a significant difference between the TPLE group and the salvage TPLE group. The pharyngeal constriction score was poorer in the salvage TPLE group than in the TPLE group $(\mathrm{P}<0.05)$ (Table IV).

Post-swallow oropharyngeal residue. The bolus residue rate in the oropharynx is depicted in Fig. 4. There was a significant difference in this rate between the TPLE group and the salvage TPLE group using Wilcoxon rank sum test $(\mathrm{P}=0.0263)$. However, there were outliers in the salvage TPLE group.
Table V. Comparison of velopharyngeal closure.

\begin{tabular}{lccc}
\hline Group & Inadequate & Normal & P-value \\
\hline TPLE & 5 & 39 & $>0.99^{\mathrm{a}}$ \\
Salvage TPLE & 2 & 14 & \\
Total & 7 & 53 & \\
\hline
\end{tabular}

aTPE group vs. salvage TPLE group. TPLE, total pharyngolaryngo-esophagectomy.

Velopharyngeal closure. The proportion of patients that experienced inadequate velopharyngeal closure is depicted in Table V; there was no significant difference between the TPLE group and the salvage TPLE group ( $\mathrm{P}>0.99)$.

\section{Discussion}

The present study demonstrated that pharyngeal constriction in patients who underwent salvage TPLE was more severe than that in patients who underwent TPLE, and that the pharyngeal residue rate of patients who underwent salvage TPLE was higher than that of patients who underwent TPLE.

Ward et al (22) demonstrated that there was a high incidence of dysphagia at discharge and during long-term follow-up in laryngectomy and pharyngolaryngectomy groups. Previous studies have shown that the major complications of CCRT are xerostomia, reduced mobility of the tongue base, reduced mobility of the larynx, reduced sensitivity (incomplete protection of the airway) and trismus (7,8). Eisbruch et al (23) demonstrated that conformal radiation reduced the radiation dose to the pharyngeal constriction. The present study revealed that the pharyngeal constriction scores of the patients who underwent salvage TPLE were poorer than those of patients who underwent TPLE. This result indicates that the adverse effects of CCRT affect pharyngeal constriction following TPLE. However, it is possible that the range of resection, reconstruction method, and the scar and edema (skin and pharyngeal mucosa) from surgery also affected swallowing function following TPLE. The present study was retrospective, so the background characteristics of the patients were not stratified, and other factors may also have affected this result. Further, the current study only evaluated the movement of the pharynx and did not evaluate pressure. It is possible that postoperative edema also affected this result.

With regards to pharyngeal residue, there was a significant difference between the TPLE group and the salvage TPLE group. The residue rate of the salvage TPLE group was higher than that of the TPLE group. Pharyngeal constriction in the salvage TPLE group may have been reduced by the initial CCRT treatment. However, there were outliers in the results of the current study, which may have affected this result. Furthermore, only the first VF examination following surgery was evaluated. It is possible that the residue rate may change with recovery, as the progress of recovery may differ for each patient. Post-swallow residue should be evaluated several times during the postoperative period. 
With regards to inadequate velopharyngeal closure, there was no significant difference between the salvage TPLE group and the TPLE group. It was possible that the range of resection was not suitably wide in the present study. If the upper resection margin was over the tonsil and included soft palate resection, soft palate movement may be limited, causing inadequate velopharyngeal closure. In the present study, the bolus flowing backward in the velopharynx was defined as a positive finding, so when the amount of bolus flowing backward in the velopharynx was too small to bed captured and objectively analyzed, the reflex may not be captured correctly.

There were limitations to the present study. Since the current study was retrospective, the number of patients in the salvage TPLE group and the TPLE group may not have been adequate for the appropriate analysis of the differences. Particularly in the TPLE group, there were a number of ineligible patients, who lacked VF records; thus, the present study may be biased as a result. The quality of life of the patients was not evaluated, and it is possible that VF findings do not associate with the subjective perceptions of patients. Finally, the present study measured only motor response; the pressure of constriction, and pharyngeal and laryngeal sensation were not analyzed. In a future study, the pressure of constriction should be evaluated directly, and the associations between the findings of examinations and the subjective perceptions of patients will require evaluation.

However, the results of thecurrent study indicated the importance of pharyngeal constriction following TPLE. Stokely et al (24) reported that pharyngeal constriction was associated with pharyngeal residue; however, pharyngeal constriction was not the only factor involved in explaining pharyngeal residue. Treatments that improve pharyngeal constriction should result in less residue. It is possible that rehabilitation, bringing about improvements such as reduced pharyngeal constriction, can reduce pharyngeal residue. Thus, rehabilitation following TPLE, such as tongue-strengthening exercises and the Masako maneuver (25) may improve swallowing function and the patient's quality of life. Therefore, a future study should also evaluate the effect of rehabilitation.

In conclusion, the swallowing function of patients who underwent salvage TPLE may be affected by CCRT. Pharyngeal constriction may be due to complications of CCRT. The results of the current study may indicate that pharyngeal constriction has a crucial role in swallowing ability following TPLE.

\section{Acknowledgements}

We are grateful to Dr Fujimoto (Department of Otorhinolaryngology, Nagoya University Graduate School of Medicine) for helpful discussions.

\section{Funding}

No funding was received.

\section{Availability of data and materials}

The datasets analyzed during the current study are available from the corresponding author on reasonable request.

\section{Authors' contributions}

TM designed the study, and wrote the initial draft of the manuscript. TM contributed to analysis and interpretation of data, and assisted in the preparation of the manuscript. All other authors contributed to data collection and interpretation, and critically reviewed the manuscript. All authors approved the final version of the manuscript, and agree to be accountable for all aspects of the work in ensuring that questions related to the accuracy or integrity of any part of the work are appropriately investigated and resolved.

\section{Ethics approval and consent to publish}

The present study was approved by the Research Ethics Committee of the National Cancer Research Center Hospital East, Chiba, Japan (No. 2016-380), and the research outline is open to the public.

\section{Consent for publication}

All patients have consented to the publication of their data.

\section{Competing interests}

The authors declare that they have no competing interests.

\section{References}

1. Adelstein DJ, Li Y, Adams GL, Wagner H Jr, Kish JA, Ensley JF, Schuller DE and Forastiere AA: An intergroup phase III comparison of standard radiation therapy and two schedules of concurrent chemoradiotherapy in patients with unresectable squamous cell head and neck cancer. J Clin Oncol 21: 92-98, 2003.

2. Forastiere AA, Goepfert H, Maor M, Pajak TF, Weber R, Morrison W, Glisson B, Trotti A, Ridge JA, Chao C, et al: Concurrent chemotherapy and radiotherapy for organ preservation in advanced laryngeal cancer. N Engl J Med 349: 2091-2098, 2003.

3. Lee SC, Shores CG and Weissler MC: Salvage surgery after failed primary concomitant chemoradiation. Curr Opin Otolaryngol Head Neck Surg 16: 135-140, 2008.

4. Esteller E, Vega MC, López M, Quer M and León X: Salvage surgery after locoregional failure in head and neck carcinoma patients treated with chemoradiotherapy. Eur Arch Otorhinolaryngol 268: 295-301, 2011.

5. Richey LM, Shores CG, George J, Lee S, Couch MJ, Sutton DK and Weissler MC: The effectiveness of salvage surgery after the failure of primary concomitant chemoradiation in head and neck cancer. Otolaryngol Head Neck Surg 136: 98-103, 2007.

6. Weber RS, Berkey BA, Forastiere A, Cooper J, Maor M, Goepfert H, Morrison W, Glisson B, Trotti A, Ridge JA, et al: Outcome of salvage total laryngectomy following organ preservation therapy: The Radiation Therapy Oncology Group trial 91-11. Arch Otolaryngol Head Neck Surg 129: 44-49, 2003.

7. Ozawa K, Fujimoto $\mathrm{Y}$ and Nakashima T: Changes in laryngeal sensation evaluated with a new method before and after radiotherapy. Eur Arch Otorhinolaryngol 267: 811-816, 2010.

8. van der Molen L, van Rossum MA, Ackerstaff AH, Smeele LE, Rasch CR and Hilgers FJ: Pretreatment organ function in patients with advanced head and neck cancer: Clinical outcome measures and patients' views. BMC Ear Nose Throat Disord 9: 10, 2009.

9. Crary MA and Glowasky AL: Using botulinum toxin A to improve speech and swallowing function following total laryngectomy. Arch Otolaryngol Head Neck Surg 122: 760-763, 1996.

10. Torres WE, Fibus TF, Coleman JJ III, Clements JL Jr and Bernardino ME: Radiographic evaluation of the free jejunal graft. Gastrointest Radiol 12: 226-230, 1987. 
11. Edge SB, Byrd DR, Compton CC, Fritz AG, Greene FL and Trotti A (eds). AJCC cancer staging manual (7th edition). New York, NY, Springer, 2010

12. Gambee LP: A single-layer open intestinal anastomosis applicable to the small as well as the large intestine. West J Surg Obstet Gynecol 59: 1-5, 1951

13. Sarukawa S, Sakuraba M, Kimata Y, Yasumura T, Uchiyama K, Hishinuma S, Nakatsuka T, Hayashi R, Ebihara S and Harii K: Standardization of free jejunum transfer after total pharyngolaryngoesophagectomy. Laryngoscope 116: 976-981, 2006.

14. Ueda K, Okada N, Kitazumi H, et al: VF evaluation criteria of the Japanese Society of Dysphagia Rehabilitation. Jap J Dysphagia Rehabil 15: 76-95, 2011

15. Dejaeger E, Pelemans W, Ponette E and Joosten E: Mechanisms involved in postdeglutition retention in the elderly. Dysphagia 12 63-67, 1997.

16. Kendall KA and Leonard RJ: Pharyngeal constriction in elderly dysphagic patients compared with young and elderly nondysphagic controls. Dysphagia 16: 272-278, 2001.

17. Leonard R, Belafsky PC and Rees CJ: Relationship between fluoroscopic and manometric measures of pharyngeal constriction: The pharyngeal constriction ratio. Ann Otol Rhinol Laryngol 115: 897-901, 2006.

18. Leonard R, Rees CJ, Belafsky P and Allen J: Fluoroscopic surrogate for pharyngeal strength: The pharyngeal constriction ratio (PCR). Dysphagia 26: 13-17, 2011
19. Yip H, Leonard R and Belafsky PC: Can a fluoroscopic estimation of pharyngeal constriction predict aspiration? Otolaryngol Head Neck Surg 135: 215-217, 2006.

20. Lan Y, Xu G, Dou Z, Lin T, Yu F and Jiang L: The correlation between manometric and videofluoroscopic measurements of the swallowing function in brainstem stroke patients with Dysphagia. J Clin Gastroenterol 49: 24-30, 2015

21. Rademaker AW, Pauloski BR, Logemann JA and Shanahan TK: Oropharyngeal swallow efficiency as a representative measure of swallowing function. J Speech Hear Res 37: 314-325, 1994.

22. Ward EC, Bishop B, Frisby J and Stevens M: Swallowing outcomes following laryngectomy and pharyngolaryngectomy. Arch Otolaryngol Head Neck Surg 128: 181-186, 2002.

23. Eisbruch A, Kim HM, Feng FY, Lyden TH, Haxer MJ, Feng M, Worden FP, Bradford CR, Prince ME, Moyer JS, et al: Chemo-IMRT of oropharyngeal cancer aiming to reduce dysphagia: Swallowing organs late complication probabilities and dosimetric correlates. Int J Radiat Oncol Biol Phys 81: e93-e99, 2011.

24. Stokely SL, Peladeau-Pigeon M, Leigh C, Molfenter SM and Steele CM: The relationship between pharyngeal constriction and Post-swallow residue. Dysphagia 30: 349-356, 2015.

25. Logemann JA: Treatment of oral and pharyngeal dysphagia. Phys Med Rehabil Clin N Am 19: 803-816, ix, 2008. 